texture of evolutionary debate. At this stage it is impossible to come up with confident estimates of the relative significance of the different ways in which genetic stasis may be threatened or secured. That must wait until there is better understanding of how the plant genome is organized, the practical importance of the different ways in which it may be altered, as well as in what ways the consequences of somatic mutation are subject to the vagaries of development and reproduction. In the interim the author is content to alert us to the manifold possiblities and engage our sympathy for his point of view.

FORBES W. ROBERTSON 41 Braid Farm Road, Edinburgh EH10 6LE

\section{Proceedings of the Second International Conference on Quantitative Genetics. Edited by BRUCE S. WEIR, Eugene J. Eisen, Major M. Goodman and Gene Namkoong. Sunderland, Mass., U.S.A.: Sinauer Associates. 1988.763 pages. $\$ 38.50$ (paper), ISBN 0878939016 . $\$ 60.00$ (cloth), ISBN 087.8939008 .}

The so-called quantitative characters tend to be given a wide berth by most geneticists, in spite of the very important part they must play in evolution and the fact that they form the main raw material for food plant and livestock improvement programmes. Such characters are generally assumed to be under the influence of many genes of small individual effect, whose interplay is obscured in practice by the large amount of environmental variation, though an occasional major gene may be caught segregating.

Study of these genetic systems is frustratingly slow to yield significant results, and this doubtless explains why the first and second international conferences on quantitative genetics were held eleven years apart (in 1976 and 1987), and attracted only a modest 250 and 500 participants, respectively. The proceedings of the second conference, under review here, might under these circumstances have been organized more with the potential newcomer to the field in view. This could have been achieved by starting with some review articles designed to give us a clear picture of progress over the years made with crop plants, livestock and 'model' laboratory animals, followed by a not too difficult review of the progress made in mathematical theory which can be applied in these areas. But we do not get assistance of this kind, and the newcomer or tenderfoot will have to pick his way among the 55 chapters (the editors' name for the individual contributions, which range from 2 to 28 pages in length) looking for the background picture. There is often only a limited connexion between neighbouring chapters (e.g. the Booroola sheep's F gene is sandwiched between two papers on human genetics); and the large amount of mathematics distributed liberally among the papers may also cause him to run for his Hardy. However, a detailed subject index is invaluable in tracing topics, and all references are collected at the end of the book to form an excellent bibliography. A few notes from scanning both chapter headings and index will give the reader a rough idea of what the book contains and omits.

Seven papers deal specifically with human quantitative genetics, and include Smouse \& Long on the population structure of two tribes of slash and burn horticulturalists, Sing et al. on disease-associated traits, Eaves et al. on human developmental change (a model), Cloninger on alcoholism, Fulker on genetic and cultural transmission in human behaviour and Iselius on genetic epidemiology of common diseases. A number of papers consider livestock quantitative genetics and its applications: the Booroola sheep in which the $F$ gene has a marked effect on ovulation rate (Piper \& Bindon), finding genes which affect quantitative traits (Pirchner), inserting retroviruses specifically the avian leucosis virus - into the avian germ line (Crittenden \& Salter - this would certainly frighten the British egg-consumer), selection response for egg production in inbred lines (Abplanalp), genetic technology and its potential in animal breeding (articles by Bechard and by Smith), and selection in farm animals (articles by Van Vleck and by Ollivier).

Molecular genetics has yet to make a major impact on livestock, regardless of the well-known transgenic mice. In plant breeding, however, major progress seems closer, following the many years of work on Agrobacterium tumifaciens and its T plasmid, the new methods such as shooting microprojectiles coated with DNA into plant cells, and linkage analysis with RFLPs to locate useful quantitative genes. Several papers discuss some of these possibilities in a rather general way: these include Soller \& Beckmann, who invent the horrible term 'Genomic Genetics' for RFLP analysis in this context and give us a mathematical run-down on how to treat the data when we get some, Sozonov on the use of biochemical markers (gliadins, glutenins etc.) to distinguish valuable wheat and barley genotypes, Shillito on the fate of DNA introduced into plants, Dudley on identifying lines useful for improving elite single crosses in corn, and Vencovsky et al. on corn breeding studies in Brazil.

Experiments on laboratory animals get less than their fair share of attention, being only represented by Mukai on genotype-environment interaction and the maintenance of variability in Drosophila melanogaster populations, Mackay on the novel study of transposable element-induced quantiative genetic variation in Drosophila, and Frankham on exchanges in the rDNA multigene family as a source of quantitative genetic variation in Drosophila. The mouse gets hardly mentioned, as do the important long-term selection experiments on tribolium by Enfield and Drosophila by Yoo and others - these focused attention on mutation as an important source of quantitative variation (see article by Hill \& Keightley).

This by no means exhausts the range of topics 
discussed, which include genetic expression of endosperm traits (Mo Huidong), differential response to environmental stress (Baker), the genetic basis of environmental sensitivity (Jinks \& Poona), metabolic control in quantitative genetics (Dean et al.), quantitative characters in Salmonids (Gjedrem et al.), three articles on quantitative genetics in trees (Van Buijtenen; Burden; Müller-Stark \& Gregorius), and microevolution of vertebral numbers in the garter snake (Arnold).

We come finally to mathematics, theory and generalities. Kempthorns, in an overview of the field, finds the theory too simplistic and looks to help from the massive use of supercomputers; Hill \& Keightley examine in depth models for predicting response to selection and the maintenance of variation, Lande discusses the development of quantitative genetics in relation to natural selection and evolution. Mathematical teeth will be needed to cope with Henderson (progress in statistical methods since 1976), Kennedy \& Sorensen (mixed model methods for predicting genetic merit), Ratner (selection and stochastic trends), Karlin (non-gaussian models), Melchinger (means and variances in hybrid populations), Wu \& $\mathrm{Li}$ (an operational genetic theory of heterosis), Zhivotovsky (analysis of correlated characters), but they can finish with Turelli's paper on population genetic models for polygenic variation and evolution.

I hope my catalogue of topics will persuade many geneticists that they will find something of interest in this book. Molecular genetic applications may make the classical plant and animal breeding experiments redundant within a few years, and may also reduce the importance of mathematical theory. But the new techniques will not be able to control the numerous genes of small effect which, we must assume, will continue to play a major role in evolutionary and artificially induced changes in quantitative characters. And finally, the book is very well edited, by the four editors and seventy-three referees, handsomely produced and cheap in its paperback form, and, of course, there is no other book like it!

ER IC REEVE Institute of Animal Genetics, University of Edinburgh

Current Communications in Molecular Biology: Viral Vectors. Edited by Yakov GLuzMaN and STEPHEN H. Hughes. New York: Cold Spring Harbor Laboratory. 1988. 180 pages. Paper \$20.00.

This latest volume in the Current Communications in Molecular Biology series contains thirty articles describing a wide range of viral vectors. No article in the paperback-sized book is larger than seven pages and each is limited to no more than three figures. In general this makes the articles concise and to the point. As a result these articles contain the information that a practising molecular biologist requires.
The most striking feature of this book is the lack of any article devoted to small DNA viruses SV40, Polymer or BK. These viruses still have uses as vectors, particularly in stable transfection systems with dominant markers and transient assays; and SV40 is probably still the most suitable vector when combined with a COS cell system for the rapid analysis of gene expression. There is still room for improvement in these vectors by removing restriction sites and inserting polylinkers. One suspects that most biotechnology companies have produced their own suite of vectors for this purpose.

Two chapters on bovine papilloviruses (BPV) open the book, but it is clear from these and the introduction by Stephen Hughes that long-term application of this virus is limited by its restricted host range and its rather unpredictable habit of integrating instead of remaining extrachromosomal. Epstein-Barr virus (EBV) is an improvement on BPV, having a broader host range and greater stability. A new generation which incorporates the orilyt replication origin is described by Sugden's group. When induced, the stable copy number of approximately 50 per cell is increased by a factor of 500-1000; how this increase in copy number influences protein production is not yet known.

The major class of vectors discussed in Viral Vectors are the Herpes viruses and retroviruses. The large Herpes-like DNA viruses are likely to have a role in the development of vaccines. Pseudorabies viruses which do not infect humans could be useful for livestock; as an experiment, Post \& Thomsen report on the expression of TPA in such a vector. An increasingly important aspect of recombinant protein production is authentic post-translational modification. In this respect Baculovirus, which grows in insect cells, can be very useful: the book describes examples of biologically active ElA, EGF receptor and protein kinase $C$ produced in this system.

Retroviruses which have in the past promised much (and have been much hyped!) as a system for introducing genes into cells and animals have still not yet made their mark, despite the extremely elaborate constructions of double promoters and sophisticated packaging cell lines. Perhaps the most exciting development in animal viral vectors, described in Viral Vectors by Schelsinger et al., is the use of Sindbis Virus as a vector. This single stranded RNA virus can be manipulated to produce up to $0.5 \mathrm{mg}$ of chloramphenicol-acetyl-transferase (CAT) in $24 \mathrm{~h}$ from $10^{9}$ cells.

Plant vectors occupy three of the thirty chapters in this book. Cauliflower Mosaic Virus (CaMV) turns out to have similarities to animal retroviruses in that replication involves reverse transcription and a double-stranded DNA intermediate. However, making viral vectors is difficult due to the small amount of disposable sequences. The CaMV $35 \mathrm{~S}$ promoter and transcription terminator directs efficient expression of 